\title{
Lateral quantum wells at vicinal Au(111) studied with angle-resolved photoemission
}

\author{
A. Mugarza, ${ }^{1}$ A. Mascaraque, ${ }^{2}$ V. Repain, ${ }^{3}$ S. Rousset, ${ }^{3}$ K. N. Altmann, ${ }^{4}$ F. J. Himpsel, ${ }^{4}$ Yu. M. Koroteev, ${ }^{1,5}$ E. V. Chulkov, ${ }^{1}$ \\ F. J. García de Abajo, ${ }^{1}$ and J. E. Ortega ${ }^{1}$ \\ ${ }^{1}$ Donostia International Physics Center and Centro Mixto de Materiales CSIC/UPV, Universidad del País Vasco, \\ Paseo Manuel Lardizabal 4, 20018-San Sebastian, Spain \\ ${ }^{2}$ LURE Centre Universitaire Paris-Sud, Bât 209 D B.P. 34, 91898 Orsay Cedex, France \\ ${ }^{3}$ Groupe de Physique des Solides, CNRS, Universités Paris 6 et 7, 2 Place Jussieu, 75251 Paris Cedex 5, France \\ ${ }^{4}$ Department of Physics, University of Wisconsin-Madison, Madison, Wisconsin 53706 \\ ${ }^{5}$ Institute of Strength Physics and Materials Science, Russian Academy of Sciences, 634021, Tomsk, Russia \\ (Received 31 May 2002; revised manuscript received 19 August 2002; published 30 December 2002)
}

\begin{abstract}
Electrons at noble metal surfaces can be confined within terraces leading to one-dimensional surface states. These can be studied with angle-resolved photoemission from vicinal surfaces with regular arrays of (111)oriented terraces. Here we show the case of $\operatorname{Au}(232321)$, which is vicinal to $\mathrm{Au}(111)$ and displays $L$ $=56 \AA$ wide terraces. The surface state band appears broken up into three quantum well levels that match to those of the infinite quantum well of the same width $L$. Their parallel momentum dependent photoemission intensity allows mapping the probability density of the confined wave function in reciprocal space using angle-resolved photoemission. By Fourier transformation, their respective experimental wave functions in real space are obtained and compared to the case of the infinite quantum-well, showing excellent agreement. Final state step superlattice diffraction effects have also been observed. Finally, we observe the quenching of the characteristic spin-orbit coupling of $\mathrm{Au}(111)$ in the confinement direction. This is another indication of the one-dimensional character of the surface state, as confirmed with first order perturbation theory.
\end{abstract}

DOI: 10.1103/PhysRevB.66.245419

PACS number(s): 79.60.Bm, 73.20.At

\section{INTRODUCTION}

Vicinal surfaces with regular step arrays are natural templates for growing nanostructured solids with lowdimensional properties. ${ }^{1,2}$ They are also convenient model systems for studying the electronic properties of lateral nanostructures. ${ }^{3-7}$ First of all because the macroscopic miscut angle (deviation from the low index plane) can be chosen to tune the step superlattice constant. And also because vicinal surfaces are readily prepared and accessed by powerful surface science techniques, such as scanning tunneling microscopy/spectroscopy (STM/STS) and angle-resolved photoemission. Vicinal noble metal (111)-surfaces are particularly suitable because they have a free-electron-like surface state that scatters strongly at step edges. In vicinal $\mathrm{Au}(111)$, this scattering at steps has been observed to lead to superlattice zone-folding effects in narrow terraces and onedimensional (1D) confinement in wider terraces. ${ }^{5}$ In this work we analyze $\mathrm{Au}(232321)$, i.e., vicinal $\mathrm{Au}(111)$ with $56 \AA$ wide terraces. As previously found in the $\mathrm{Au}(887)$, the electronic structure is characterized by the presence of nondispersing energy levels in the direction perpendicular to the steps, indicating total electron confinement within terraces. The wave functions of the first three quantum well $(\mathrm{QW})$ levels are directly probed in reciprocal space by angleresolved photoemission, in a similar way as it is done by STM/STS in real space. ${ }^{8,9}$ We show that a consequence of the terrace confinement is the cancellation of the surface spin-orbit coupling (SOC) in the perpendicular direction to the steps, in contrast to the isotropic SOC observed in $\mathrm{Au}(111)$. A direct theoretical description of the SOC using first order perturbation theory reproduces the cancellation of the spin-orbit splitting across the steps. The theory also shows that the full contribution to the SOC splitting comes from the potential gradient at the nuclear region of the first 2-3 layers. On the other hand, the superlattice nature of the vicinal surface shows up in the diffraction of the final state, in analogy to low-energy electron diffraction (LEED) electrons.

\section{EXPERIMENT}

The photoemission data has been acquired with a hemispherical Scienta SES200 spectrometer equipped with angle and energy multidetection and coupled to an undulator PGM beam line at the Synchrotron Radiation Center (SRC) of the UW-Madison. The energy and angular resolution were $20+7$ $\mathrm{meV}$ (photons + electrons) and $0.39^{\circ}$, respectively. The emission plane for multidetection was set perpendicular to the incidence plane of the light ( $p$ polarized), such that a complete two-dimensional map could be taken by changing the tilt, i.e., rotating the sample $\sim 12^{\circ}$ around the axis of the spectrometer. In Fig. 1(a) we schematicaly show the geometry of the photoemission experiment from a vicinal surface. Here the $(x, y, z)$ coordinates are related to the average surface plane and the $\left(x^{\prime}, y^{\prime}, z^{\prime}\right)$ to the (111) terrace plane. In this work we only show cuts from two-dimensional maps in the direction perpendicular and parallel to the steps. These two relevant directions of the surface were identified by the characteristic splitting of the LEED spots in the direction perpendicular to the steps, and cross checked by the splitting of the spectra due to diffraction by the step array. The single crystal was prepared by extensive sputtering-annealing cycles until the LEED pattern displayed a clear spot splitting. The experiments were performed with the surface held at $120 \mathrm{~K}$. 

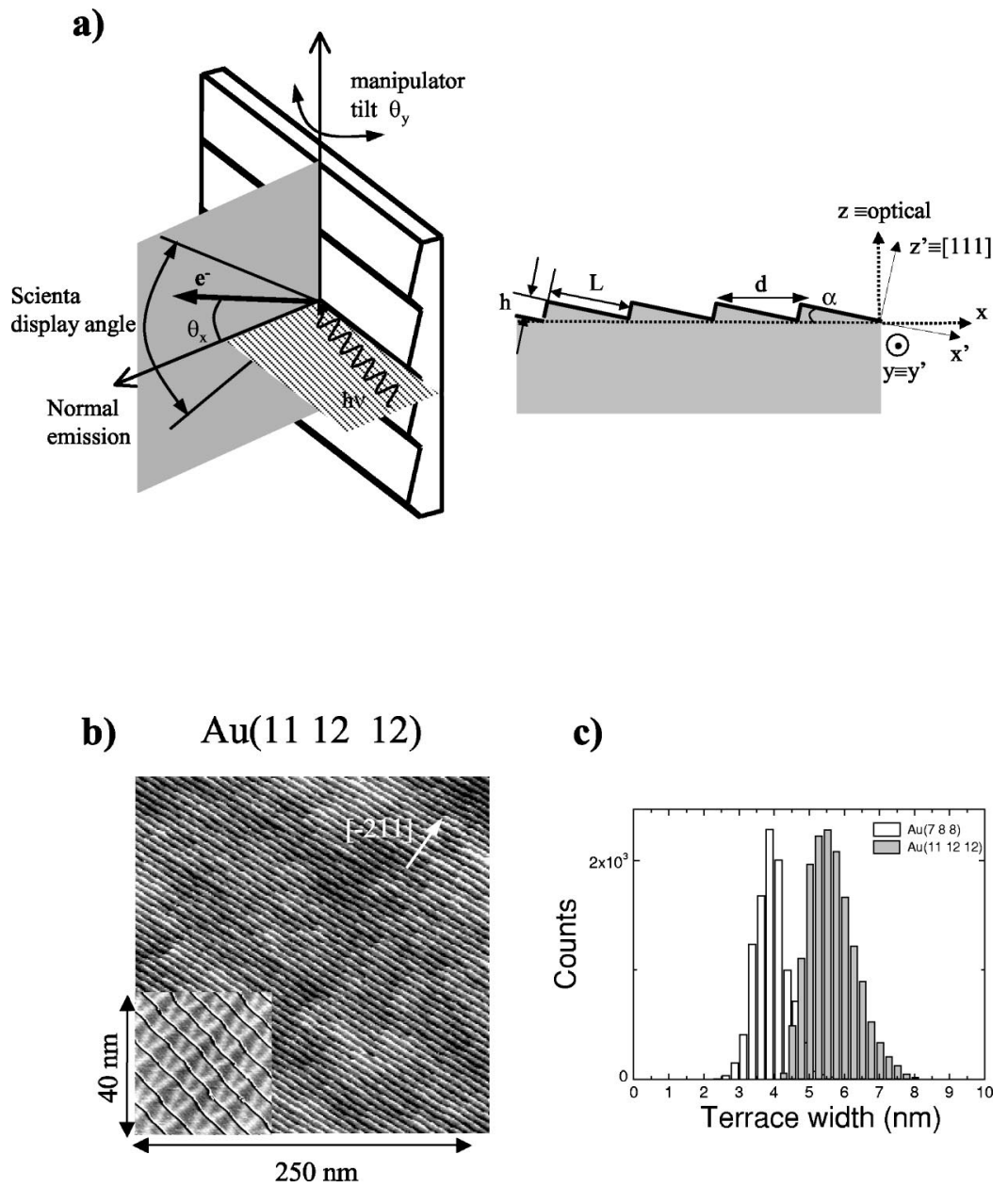

c)

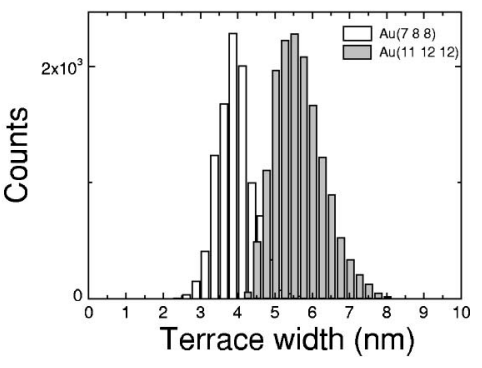

FIG. 1. (a) Geometry of the angle-resolved photoemission experiment (left) and a vicinal surface (right). The two reference systems used in this work are indicated in the right figure, i.e., $(x, y, z)$ respect to the average (optical) surface and $\left(x^{\prime}, y^{\prime}, z^{\prime}\right)$ respect to the (111) terrace. (b) STM image of $\mathrm{Au}\left(\begin{array}{lll}23 & 23 & 21\end{array}\right)$ showing straight monoatomic steps and (c) the corresponding terrace width distribution. The inset in (b) shows the detailed structure at a smaller scale. The corrugation due to the terrace levels has been subtracted in order to enhance the terrace corrugation. Bright and dark areas indicate the presence of disconmensuration lines that separate the alternating fcc- and hcp-packed layers within the same terrace (Ref. 11).
Figure 1(b) shows an STM image of $\mathrm{Au}(2323$ 21) on a large scale. This high index surface is vicinal to $\mathrm{Au}(111)$ with a miscut angle of $2.4^{\circ}$ towards $[\overline{2} 11]$. The surface is composed of monoatomic $\{111\}$-like steps that separate terraces of nominal width $L=56 \AA$. The inset shows a zoom of a few terraces where the corrugation due to the terrace levels has been subtracted. This enhances the disconmesuration lines running perpendicular to the step edges that indicate the presence of alternating fcc- and hcp-packed domains along a single terrace. Figure 1(c) displays the terrace width distribution (TWD) of this surface measured over 30 images and more than 200 terraces, which is compared to the TWD of $\mathrm{Au}(8 \quad 87)$. The resulting average terrace width values for both vicinals are $\langle L\rangle=39 \pm 4 \AA$ for $\operatorname{Au}\left(\begin{array}{lll}8 & 8 & 7\end{array}\right)$ and $\langle L\rangle=56$ $\pm 6 \AA$ for $\mathrm{Au}(232321)$, which corresponds to miscut angles of $\alpha=3.5^{\circ}$ and $\alpha=2.4^{\circ}$, respectively. The ratio of Gaussian standard deviation and mean terrace width is $\sigma /\langle L\rangle=0.11$ for both surfaces. If we compare this value to those found at different vicinal $\mathrm{Cu}$ surfaces, the latter are higher by a factor of 2 or $3 .^{10}$ This narrower terrace width distribution in $\mathrm{Au}(232321)$ is a consequence of an effective stronger repulsive step-step interaction, which in turn appears to be due to the surface reconstruction. ${ }^{11}$ It is important to remark that, the averaging character of the angle-resolved photoemission requires surfaces with very narrow TWD in the scale of the photon beam size, which is typically of the order of $0.5-1$ $\mathrm{mm}^{2}$.

\section{1D TERRACE CONFINEMENT}

In general, the step array on noble metal vicinals induces anisotropy in the surface electronic structure. ${ }^{3-7}$ The freeelectron-like dispersion along the terraces still shows no influence of the step array. However, in the perpendicular direction the terrace width determines a completely different electronic structure: the surface state dispersion appears as a band with the superlattice zone folding for narrow terraces while it splits into flat, nondispersing energy levels for the wider terraces. ${ }^{5}$ The latter is the case of $\mathrm{Au}(232321)$. In Fig. 2 the photoemission spectra near the Fermi level show the dispersion of the surface state peak parallel ( $y$ direction) and perpendicular ( $x$ direction) to the steps. The momentum parallel to the average surface is obtained from the emission angle and the measured kinetic energy with respect to the vacuum with the well-known formula

$$
k_{x, y}=q_{x, y}=\sqrt{\left(2 m / \hbar^{2}\right) E_{k i n}} \sin \theta_{x, y},
$$

where $k$ and $q$ are the momentum inside and outside the crystal, respectively. In the presence of surface umklapp the parallel momentum inside the crystal is given by $k_{x, y}=q_{x, y}$ $+g_{x, y}$, where $g_{x, y}$ is a surface lattice vector. Such surface 

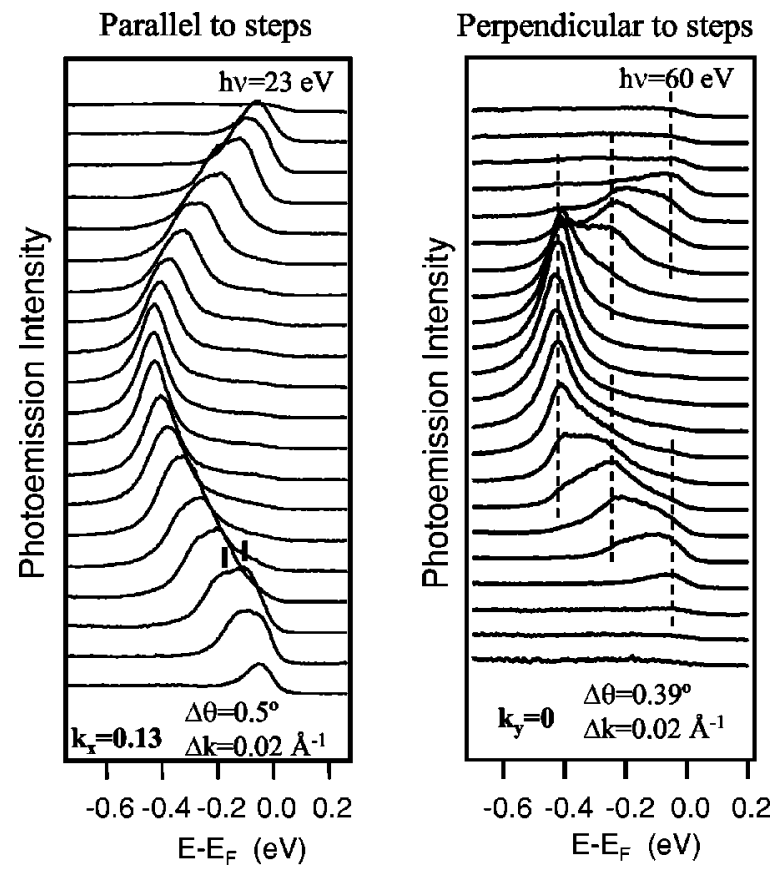

FIG. 2. Dispersion of the surface state at $\mathrm{Au}(232321)$ parallel (left) and perpendicular (right) to the step superlattice. The strong anisotropy indicates the presence of a one-dimensional surface state, i.e., a free-electron-like state parallel to the steps, but confined in the perpendicular direction, where the free-electron-like parabola splits in three different quantum well levels. These are marked with dashed lines. The ticks in the left figure indicate the spin-orbit split peaks in the direction parallel to the steps.

umklapps are observed in our experiment, as we show later. In order to avoid these umklapp features, a photon energy of $60 \mathrm{eV}$ was used for the energy analysis of the nondispersing features in the direction perpendicular to the steps. On the other hand, the direction parallel to the surface does not show any umklapp and thus a lower photon energy of $23 \mathrm{eV}$ was used to maximize the energy resolution. The different dispersing behavior along the $x$ and $y$ directions is already observed in Fig. 2. The dispersion parallel to the steps is identical to the flat $\mathrm{Au}(111)$ surface, i.e., free-electron-like band and momentum dependent splitting due to the surface SOC (marked with ticks in the figure). In contrast, the spectra perpendicular to the steps show three nondispersing energy levels, indicating electron confinement along this direction. Similar 1D quantum well levels have been also

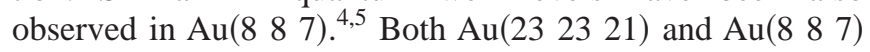
are already in the large terrace width regime, where $1 \mathrm{D}$ confinement of the surface electrons takes place. At a smaller terrace width $\left(L_{c} \simeq 15 \AA\right)$, a transition to a 2D superlattice state is expected. ${ }^{5}$ On the other hand, no spin-orbit splitting is observed within the energy resolution in the perpendicular direction.

The photoemission intensity of the three QW levels is clearly modulated along the direction perpendicular to the steps. This can be better observed in Fig. 3(a), where a gray scale plot of the second derivative of the raw data is shown together with the data for $\mathrm{Au}(8 \mathrm{8} 7)$. The second derivative of the EDC curves can be roughly taken as proportional to the ratio between the intensity $I$ and the width $W$ of the peaks $I / W .^{12}$ Since the width of the QW levels do not show any significant change, we can directly compare these derivatives and the photoemission intensity. Thus, the gray scale plot of Fig. 3(a) represents the three QW levels and their angulardependent intensity. The dashed lines correspond to the average of the peak energies obtained by line-fitting the individual spectra of Fig. 2 right [the intensity maxima of the $N=2$ level in Fig. 3(a) right is slightly shifted from the average value in the fitting due to the effect of the Fermi edge on the second derivative]. Note that the intensity maxima of the QW levels intersect the free-electron-like band of the flat, infinite terrace width surface. This is analogous to $2 \mathrm{D} \mathrm{QW}$ energy levels tracking bulk bands in thin films.

In Fig. 3(b), the energies obtained by fitting the EDC

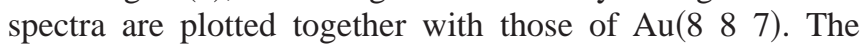
fitting was done with three Lorentzians after substraction of a Shirley and a smooth background, all convoluted with a Gaussian in order to account for the overall experimental resolution. The three energy levels $(-0.420,-0.250$, and $-0.055 \mathrm{eV})$ match very well with the levels corresponding to the infinite $\mathrm{QW}$ of the same width $L=56 \AA$, as shown in Fig. 3(b). The energy of the Nth level for the infinite QW is referred to $E_{F}-E_{0}$, i.e., the ground state of the surface with a terrace of infinite width $(L=\infty)$ :

$$
E_{N}=E_{F}-E_{0}+\frac{\hbar^{2} \pi^{2}}{2 m^{*} L^{2}} N^{2}
$$

Fitting the energy levels of $\mathrm{Au}(232321)$ with Eq. (2), and taking the reference energy as the only parameter we obtain $E_{F}-E_{0}=-0.460 \mathrm{eV}$, whereas in $\mathrm{Au}(8 \quad 8$ 7) the reference energy found was $E_{F}-E_{0}=0.5 \mathrm{eV}$. Both values lie close to the minimum of the surface state band at $\mathrm{Au}(111)$ found at the literature, which varies between -0.5 and $-0.40 \mathrm{eV},{ }^{13-16}$ depending on the particular measuring conditions, such as temperature, energy and angular resolution. However, we cannot discard physical reasons to explain a lower reference energy found for narrower terraces, such as a lower density of defects. In Fig. 3(b) we choose the reference energy of $E_{F}-E_{0}=-0.480 \mathrm{eV}$ to simultaneously fit the energy levels of both surfaces.

The QW peak energies obtained in the fitting have a standard deviation of $\sim 15 \mathrm{meV}$ for the first level and $\sim 35 \mathrm{meV}$ for the second level. The third level appears to be cutoff by the Fermi level. Such broadening could be either attributed to the finite TWD or to a finite transmission through the steps thus leading to a residual dispersion of the superlattice subbands. To estimate the maximum transmission probability we make use of the 1D Kronig-Penney (KP) model with $\delta$-Dirac potentials and potential barrier $U_{0} a$, i.e., $V(x)=\Sigma_{n} U_{0} a \delta(x$ $-n d)$. Fitting our $E(k)$ data to KP subbands we can obtain a value for the minimum potential barrier $U_{0} a$, which is found to be $10 \mathrm{eV} \times \AA$ for $\mathrm{Au}(232321)$. For $\mathrm{Au}(8 \mathrm{8}$ 7) we find a significatively higher value of $25 \mathrm{eV} \times \AA$. This corresponds to maximum transmission probabilities $|T|_{\max }^{2}$ of 0.18 and 0.04 , respectively, at $E-E_{F}=-0.1 \mathrm{eV}$, the energy where we find a QW level at both surfaces [see Fig. 3(b)]. 
a)

$\operatorname{Au}(232321)$
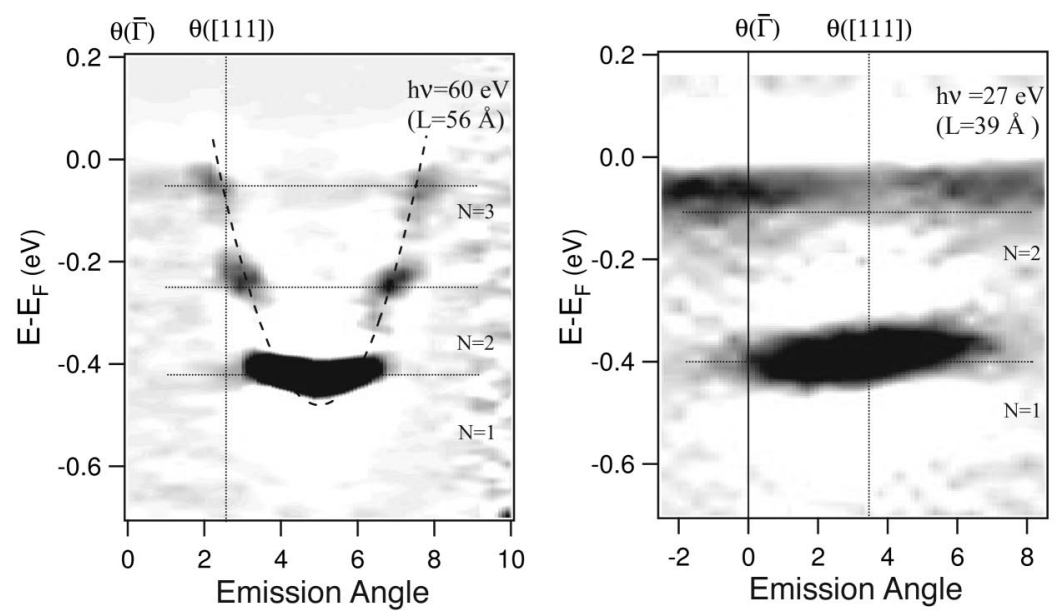

b)

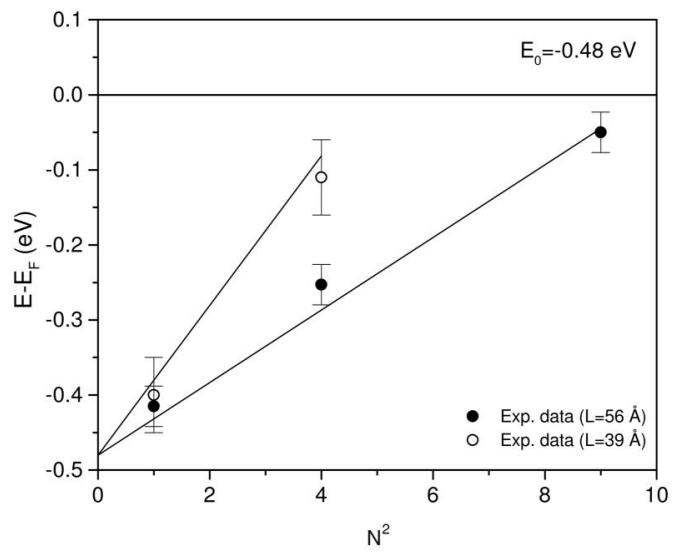

These differences found in the potential barriers of the two surfaces are not expected for vicinals with similar miscut angle. Moreover, the peak energies obtained in the fitting do not show any sign of zone folding as an indication of a superlattice state originated by the step array. On the other hand, the TWD would lead to an energy broadening of the QW levels. For $N=1$ and $N=2$ in $\mathrm{Au}(232321)$, and with our experimental TWD, this amounts to $\Delta E=10 \mathrm{meV}$ and $\Delta E=39 \mathrm{meV}$, respectively. These values are similar to the standard deviation of the peak energies of each level obtained in the fitting. In addition, the overall energy and momentum broadening in the presence of a finite TWD is expected to give rise to a paraboliclike intensity modulation, such as the one observed in Fig. 3(a). Thus, we conclude that, although some small finite transmission effects could be hidden by the energy broadening coming from the TWD, the comparison of the energy levels with the infinite QW levels indicate total confinement, i.e., $|T|^{2} \sim 0$.

This result agrees with the STM experiments done on single terraces for $\mathrm{Au}(111)$ and $\mathrm{Ag}(111))^{8,9,17}$ Experiments performed in $\mathrm{Cu}(111)$ by creating quantum corrals with $\mathrm{Fe}$ adsorbates ${ }^{18,19}$ and further theoretical work ${ }^{20}$ suggest similar reflection properties as in $\mathrm{Au}$ and $\mathrm{Ag}$, i.e., low transmission through the scatterers. The relatively low reflectivity is ex-
FIG. 3. (a) Gray scale plot of the second energy derivative of the spectra for $\mathrm{Au}(232321)$ and $\mathrm{Au}\left(\begin{array}{l}8 \\ 8\end{array} 7\right)$. The black color represents the QW state peaks, which appear to be modulated in intensity along the emission angle. The lack of dispersion of these levels indicates total confinement. The average of the energy levels obtained by fitting individual spectra are represented by dashed lines. The dotted lines indicate the (111) direction. (b) Fitting of the QW levels with the 1D Kronig-Penney model taking the reference energy as the only parameter. For finite potentials, we relate the average energy of the $N$ th band with the energy of the $N$ th level of the infinite well. The best fitting for both vicinals is obtained with infinite potential well and $E_{0}=-0.480 \mathrm{eV}$. plained in terms of absorption to the bulk, which is also inferred from the width of the quantum well levels. In contrast, an independent theoretical calculation of different adsorbates and missing rows found a higher transmission coefficient. $^{21}$ Nevertheless, none of these works referred to steps as scatterers, thus it is not so straightforward to relate their results with photoemission results on vicinal surfaces. To our knowledge, no photoemission experiments have been performed with $\mathrm{Cu}(111)$ vicinals of terrace widths comparable to the ones in this experiment. The parabolic dispersion of photoemission results for a $\mathrm{Cu}(111)$ vicinal with smaller terraces $(L=24 \AA$ ) still indicates some finite transmission, but this could be due to the higher surface state coupling to the bulk present in vicinal surfaces. ${ }^{3,5}$ It is also likely that the wider TWD found for vicinal $\mathrm{Cu}(111)$ surfaces might convolute all QW levels in an effective parabola.

\section{DIFFRACTION OF THE FINAL STATE}

In Fig. 4(a) we present a gray scale plot of photoemission spectra as a function of photon energy, where we can observe momentum splitting in the direction perpendicular to the steps. The photoemission intensity switches from left to right as we go up in photon energy. The left hand feature appears dominant at $23 \mathrm{eV}$, and completely vanishes at $60 \mathrm{eV}$. The 
a)

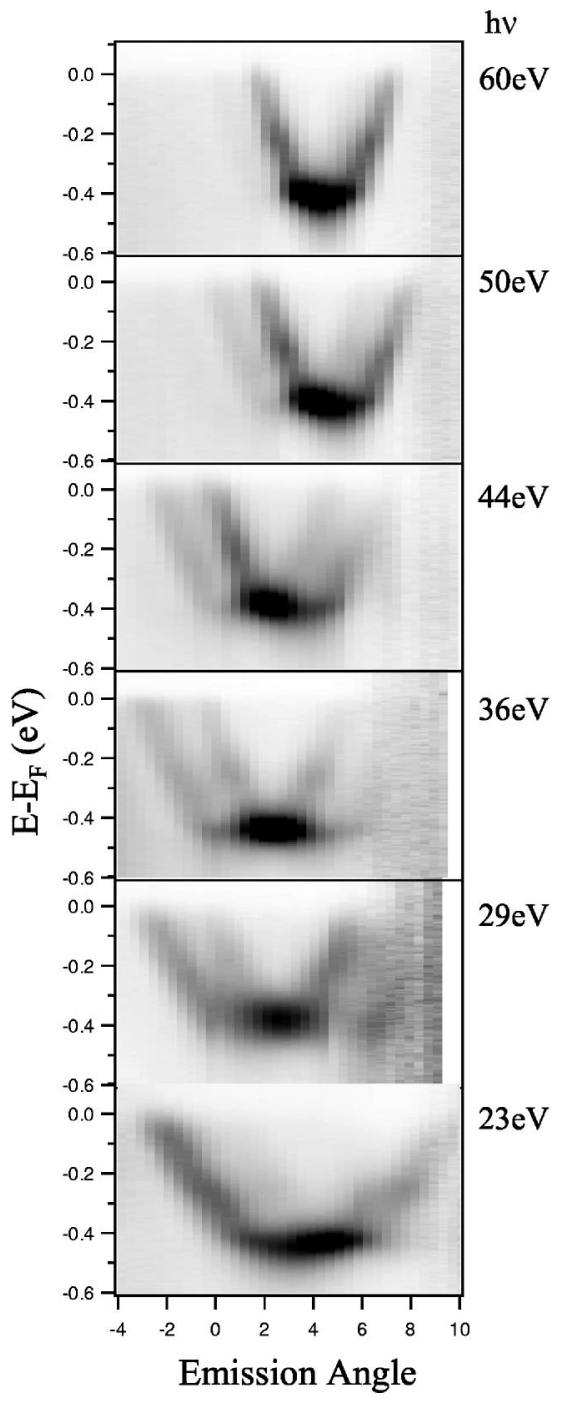

b)

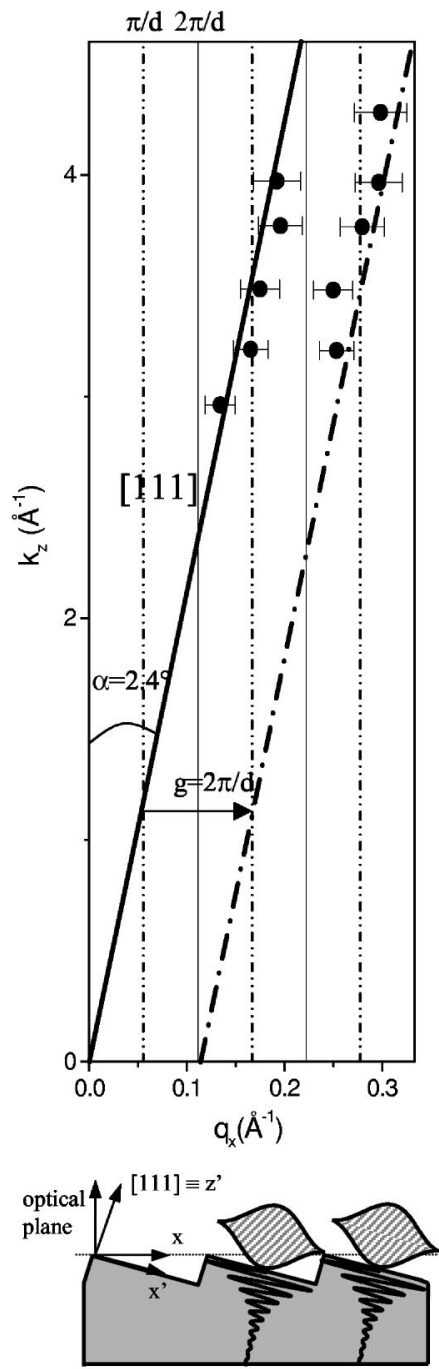

FIG. 4. (a) Dispersion in the direction perpendicular to the steps for different photon energies, showing a duplication of the parabolic envelope of QW states due to surface umklapp (b) $\left(q_{x}, q_{z}\right)$ final state wave vector components inside the crystal ( $q_{x}$ outside the crystal, see text) calculated at the center of the parabolas in (a). The reference system is the optical plane (see the figure below). Data points fall along two lines parallel to the [111] direction, which are separated by a reciprocal lattice vector $g=2 \pi / d$ of the step superlattice, as expected for step superlattice umklapp. The spectral distribution in Fig. 4(b) agrees with the real space description of the $N=1 \mathrm{QW}$ state wave function confined within [111] terraces, as shown below. overall behavior shown in Fig. 4(a) can be understood by means of the wave vector plot displayed in Fig. 4(b). Data points in this figure correspond to the electron final state momentum inside the crystal $\left(q_{x}, q_{y}=0, q_{z}\right)$ calculated for the maximum intensity point of the $N=1$ level, which is at the minimum of the parabolic envelopes in Fig. 4(a). $q_{x}$ is directly obtained from Eq. (1). Strictly speaking, $q_{x}$ is the parallel momentum outside the crystal but, as said in Sec. III, it can differ from the momentum inside the crystal just by a superlattice wave vector $g$. To a first approach, $q_{z}$ can be derived from free-electron-like final bands by invoking energy conservation to obtain the following expression:

$$
q_{z}=\sqrt{\left(2 m / \hbar^{2}\right)\left(E_{\mathrm{kin}}+V_{0}\right)-q_{x}^{2}},
$$

where $V_{0}=15.2 \mathrm{eV}$ represents the potential step at the surface. $^{22}$ Data points line up along the (111), $z^{\prime}$ direction, which is represented in the figure by a solid line. This is a consequence of a broad momentum distribution of the initial state along the (111) direction that arises from the confinement of the electron within the terrace plane. ${ }^{3}$ We can also observe a second alignment in the same direction but shifted in $q_{x}$ (dashed line). The average separation between the two sets of data points is $0.10 \AA^{-1}$, which is very close to the step superlattice wave vector $g=2 \pi / d=0.11 \AA^{-1}$, suggesting a surface umklapp process. This umklapp can only come from the final state, since the QW state is confined within a single terrace and therefore cannot be diffracted by the periodic potential of the step superlattice. Therefore, we can say that the zero $\left(g_{0}\right)$ and first diffracted order $\left(g_{1}\right)$ of the superlattice are observed in the photoemission spectra.

In principle the diffraction of nonlocalized photoelectrons should be identical to diffraction in LEED. In order to check this analogy we can use the kinematical approximation to derive the following simple relations for the in-phase (singlet) and anti-phase (doublet) conditions of the (00) LEED spot in a vicinal surface: ${ }^{23}$

$$
E_{(00)}=\frac{150 s^{2}}{4 d^{2}}[\mathrm{eV}],
$$

where $s$ is an integer (half-integer) for in-phase (antiphase) condition and $d$ is the step height in $\AA$. This equation holds for normal incidence and backscattering referred to the (111) 
a)

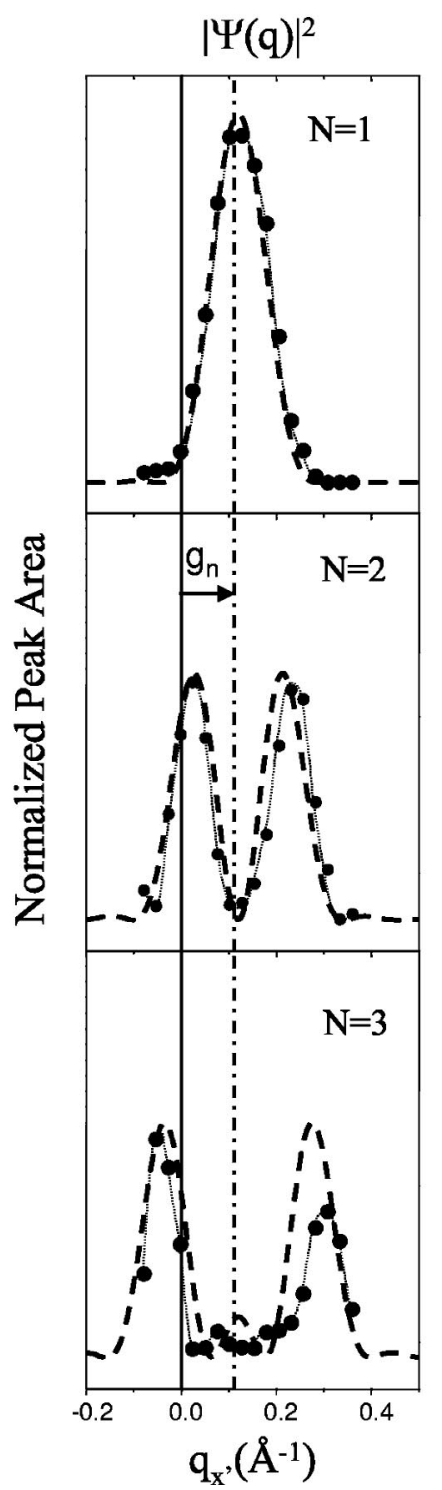

b)

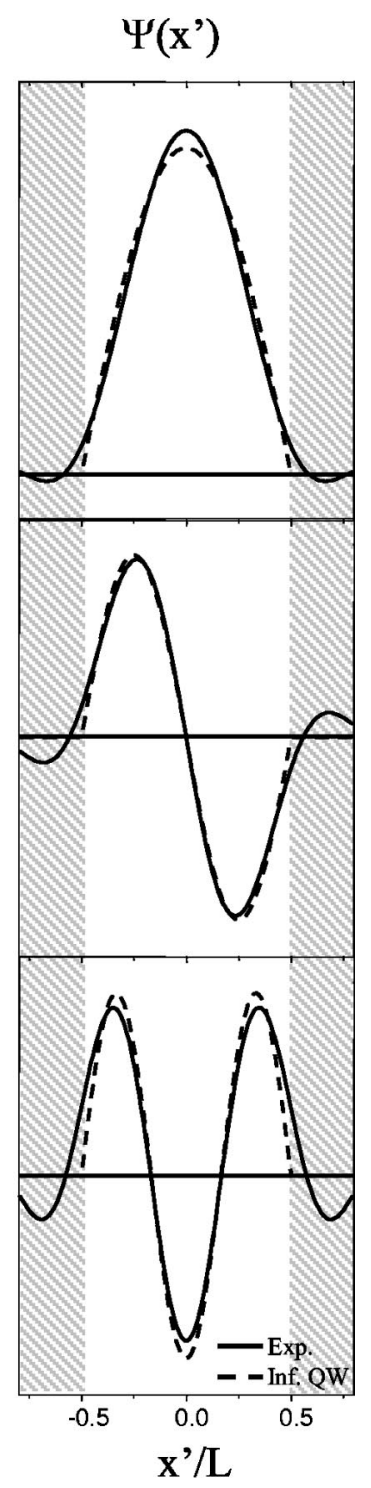

FIG. 5. (a) Intensity of the three quantum levels as a function of the wave vector parallel to the terrace and perpendicular to the steps. The thin dotted lines are interpolation curves of the data points. The dashed lines represent the expected photoemission intensity from the infinite QW of the same width. The $q_{x^{\prime}}=0,2 \pi / d$ points are marked with lines. (b) Wave functions corresponding to the photoemission intensities in (a). The solid lines correspond to the experimental wave functions (see text), and their respective infinite QW wave functions are represented with dashed lines. terraces, which is the emission direction of the maximum intensity point of the $N=1$ level that we are comparing. In the energy range of our photoemission experiments, two energies for in-phase condition are found at 27 and $61 \mathrm{eV}$, and one antiphase condition at $42 \mathrm{eV}$. In order to compare this values with the photoemission diffraction, we can obtain the kinetic energy of the photoelectron at the Fermi level from the relation $E_{\text {kin }}=h \nu-\phi$, where $\phi=5.3 \mathrm{eV}$ is the work function of $\mathrm{Au}(111)$. In Fig. 4(a) we see single order spectra at kinetic energies of $\sim 18$ and $55 \mathrm{eV}$, similar to the kinetic energies for in-phase condition in LEED.

\section{MAPPING THE WAVE FUNCTION}

From the fitting to the EDC curves we can obtain the angular modulation of the photoemission intensity shown in Fig. 5(a). In a previous work, ${ }^{5}$ a formalism was presented where a direct relation between the angle dependent photoemission intensity and the square modulus of the probability density in reciprocal space was set. In this work, the importance of including final state effects was remarked in order to obtain a more complete description of the angular dependence of the photoemission matrix element. In the derivation presented here, a more realistic final state is included which takes into account the diffraction by the step superlattice.

The angular dependence of the peak area for each quantum level is directly proportional to the angle-resolved photoemission matrix element, which in first order perturbation can be expressed as

$$
I \propto\left|\left\langle\psi_{i}|\mathbf{A} \cdot \mathbf{p}| \psi_{f}\right\rangle\right|^{2} .
$$

Here $\mathbf{A}$ and $\mathbf{p}$ are the electromagnetic vector potential and electron momentum operators and $\psi_{i}$ and $\psi_{f}$ the wave func- 
tions of the initial and final states, respectively. The initial and final wave functions can be written in the following way:

$$
\psi_{i}\left(x^{\prime}, y^{\prime}, z^{\prime}\right)=e^{i k_{y^{\prime}} y^{\prime}} \phi\left(x^{\prime}\right) \varphi\left(z^{\prime}\right)
$$

where

$$
\varphi\left(z^{\prime}\right)= \begin{cases}A_{v} e^{-\lambda_{v} z^{\prime}}, & z^{\prime}>0, \\ A_{c} e^{\lambda_{c} z^{\prime}} \cos \left(k_{z^{\prime}} z^{\prime}+\delta_{z^{\prime}}\right), & z^{\prime}<0,\end{cases}
$$

and

$$
\psi_{f}\left(x^{\prime}, y^{\prime}, z^{\prime}\right)=\sum_{n} \alpha_{n} e^{i q_{z^{\prime}} z^{\prime}} e^{i q_{y^{\prime}} y^{\prime}} e^{i\left(q_{x^{\prime}}-g_{n}\right) x^{\prime}},
$$

where the superlattice wave vector $g_{n}=(2 \pi / d) \times n$ accounts for the diffraction of the final state by the step array, and the $\left(x^{\prime}, y^{\prime}, z^{\prime}\right)$ system is referred to the terrace plane, as shown in Fig. 1(a). With these wave functions, since our experimental geometry implies grazing incidence and $p$-polarized light $\left(\mathbf{A} \approx A_{z^{\prime}} \hat{z}^{\prime}\right)$, Eq. (5) can be written as

$$
\begin{aligned}
I\left(q_{x^{\prime}}, q_{y^{\prime}}\right) \propto & A_{z^{\prime}}^{2}\left|\left\langle\varphi\left(z^{\prime}\right)\left|p_{z^{\prime}}\right| e^{i q_{z^{\prime}} z^{\prime}}\right\rangle\right|^{2} \\
& \times\left|\left\langle\phi\left(x^{\prime}\right) \mid \sum_{n} \alpha_{n} e^{i\left(q_{x^{\prime}}-g_{n}\right) x^{\prime}}\right\rangle\right|^{2} \\
& \times\left|\left\langle e^{i k_{y^{\prime} y^{\prime}}} \mid e^{i q_{y^{\prime}} y^{\prime}}\right\rangle\right|^{2} \\
= & C \delta\left(k_{y^{\prime}}-q_{y^{\prime}}\right)\left|\left\langle\phi\left(x^{\prime}\right) \mid \sum_{n} \alpha_{n} e^{i\left(q_{x^{\prime}}-g_{n}\right) x^{\prime}}\right\rangle\right|^{2} \\
& \left.\propto \sum_{n} \alpha_{n} \mid \widetilde{\phi}_{(} q_{x^{\prime}}-g_{n}\right)\left.\right|^{2},
\end{aligned}
$$

where $C$ is constant and the $\delta\left(k_{y^{\prime}}-q_{y^{\prime}}\right)$ accounts for the momentum conservation in the $y^{\prime}$ direction. In our experiment the emission is set in the $x^{\prime} z^{\prime}$ plane, thus $q_{y^{\prime}}=0$ and $I\left(q_{x^{\prime}}, q_{y^{\prime}}\right) \equiv I\left(q_{x^{\prime}}\right)$. At the photon energies used in the experiment and close to the surface normal $q_{z^{\prime}}\left(q_{z^{\prime}}\right.$ $\left.\gg q_{x^{\prime}}, q_{y^{\prime}}\right)$ is fixed by energy conservation so that the $z^{\prime}$ term remains approximately constant for a small emission angle range. Strictly speaking, the step superlattice direction is defined in the direction along the optical plane but for small miscut angles and for large $q_{z^{\prime}}$, we can suppose superlattice momentum transfers in the $x^{\prime}$ direction. Thus, the photoemission cross section only depends on $q_{x^{\prime}}$ and is proportional to a linear combination of functions which represent the 1D probability density in reciprocal space shifted by $g_{n}$, the reciprocal superlattice vector. This model could be extended to a 2D nanostructure without any loss of validity. ${ }^{24}$ As seen in the previous section, by varying the photon energy, we select different diffraction orders. In Fig. 5 we show the case of $60 \mathrm{eV}$ photon energy, which shows single diffracted order spectra. In such a case Eq. (9) reduces to

$$
I\left(q_{x^{\prime}}\right) \propto\left|\widetilde{\phi}\left(q_{x^{\prime}}-g_{n}\right)\right|^{2} .
$$

In the case of the infinite QW, Eq. (10) would take the following simple form:

$$
I_{\mathrm{QW}_{\infty}}\left(q_{x^{\prime}}\right)=A \times \frac{1-(-1)^{N} \cos \left[\left(q_{x^{\prime}}-g_{n}\right) L\right]}{\left[\left(q_{x^{\prime}}-g_{n}\right)^{2}-\left(\frac{\pi N}{L}\right)^{2}\right]^{2}} \times N^{2} .
$$

By fitting the experimental intensity curves with Eq. (11) we get $g=0.12 \AA^{-1}$, very similar to the first order superlattice wave vector $g_{1}=2 \pi / d=0.11 \AA^{-1}$. In Fig. 5(a) data are compared with the photoelectron intensity of an infinite QW, showing very good agreement. Note that $I\left(q_{x^{\prime}}\right)$ in Eq. (10) actually represents the square of the Fourier transform of the wave function shifted by a superlattice wave vector. Thus, after the correction of this shift, we can obtain the probability density in real space by inverse Fourier transformation of the experimental data. The result should be comparable to the densities obtained by STM. The only unknown parameter for the determination of the wave function is the phase $\delta(q)$ in momentum space. To a first approach, we can fix $\delta(q)$ by assuming a symmetric, real terrace potential, and thereby forcing the wave functions to be also real and either odd or even. Then, the phase is defined by the following expression:

$$
\delta(q)= \begin{cases}0, \pi, & \psi(x) \text { even, } \\ \pm\left[\frac{\pi}{2}-\pi \Theta(q)\right], & \psi(x) \text { odd, }\end{cases}
$$

where $\Theta(q)$ is the step function. The wave function then becomes

$$
\psi\left(x^{\prime}\right)=\int e^{i q_{x^{\prime} x^{\prime}}} \sqrt{I\left(q_{x^{\prime}}\right)} e^{i \delta(q)} d q_{x^{\prime}} .
$$

The resulting experimental wave functions are shown in Fig. 5(b) and compared to the case of the infinite well. The agreement is very good. The slight penetration observed in the experimental wave functions could be an indication of the finite value of the potential well or, in other words, a slightly wider effective terrace width. In any case, such a finite penetration does not lead to any energy dispersion within our energy resolution.

\section{SPIN-ORBIT COUPLING AT TERRACES}

In the free-electron-like dispersion parallel to the terraces shown in Fig. 2 we can observe the splitting of the surface state peak due to the spin-orbit interaction. In Fig. 6 we show the second derivative of these spectra displayed in gray scale for the momentum parallel to the steps, together with the fitted parabolas. As follows from Fig. 6 the free-electron-like parabola splits in two, as observed for the surface state on the flat $\mathrm{Au}(111)$ surface. ${ }^{14,15}$ The spectra has been fitted with two parabolas as shown in the figure with thin lines, and the values obtained for this splitting indicates no difference from the flat $\mathrm{Au}(111)$ case. The energy splitting at the Fermi level is $\Delta E \sim 0.122 \mathrm{eV}$ and the momentum splitting $\Delta k=$ $\pm 0.013 \AA^{-1}$, very similar to the values found in literature 


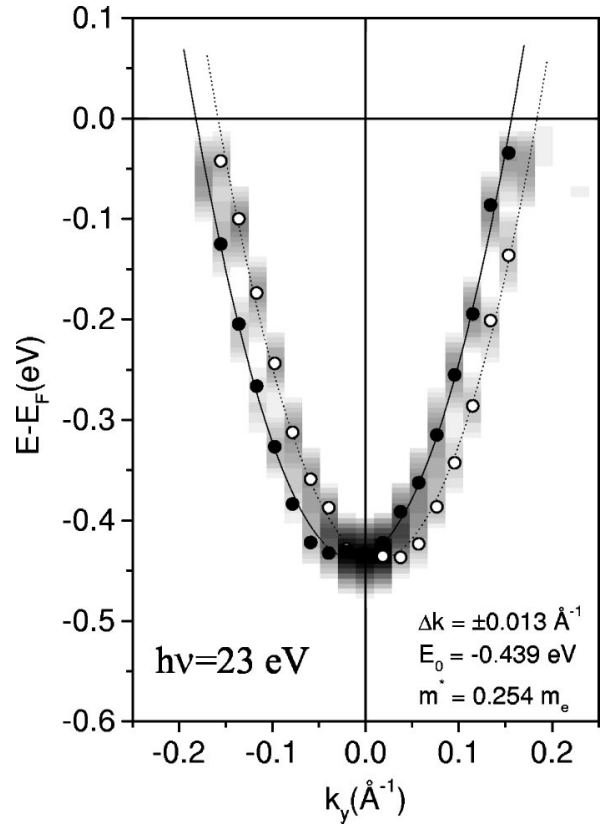

FIG. 6. Dispersion parallel to the steps, showing the spin-orbit splitting. The gray scale plot represents the second energy derivative of the raw data, the dots are the peak positions obtained from a fit and the lines are parabolic fit to these points. The Fermi crossings for the two parabolas are $k_{F}=0.157,0.184 \AA^{-1}$.

for flat $\mathrm{Au}(111) .{ }^{14,15}$ The slightly lower Fermi crossing of both parabolas observed in our case, $k_{F}=0.157,0.184 \AA^{-1}$, are due to the upward energy shift caused by the confinement in the perpendicular direction.

The spin-orbit splitting does not show up in the QW spectra perpendicular to the steps shown in Fig. 2(right), suggesting the SOC cancellation along this direction. In fact, using two split peaks does not result in any significant improvement in the fit of the QW features, neither in any reasonable $k$ dependence of the splitting. In order to explain this effect we need to deep into the origin of the SOC splitting. ${ }^{25,27}$ Retaining in the SOC term only contributions with the fastest change of one electron potential, i.e., in a region close to the nuclei and at the surface, one can write $H_{\text {SOC }}$ in the first order perturbation theory in the following way:

$$
H_{\mathrm{SOC}}=\frac{\hbar^{2}}{4 m^{2} c^{2}}\left[\frac{1}{r} \frac{d V_{\mathrm{nuc}}(r)}{d r}(\mathbf{L} \cdot \sigma)+\left[\nabla V_{\text {surface }}(\mathbf{r}) \times \mathbf{p}\right] \cdot \sigma\right],
$$

where $\sigma$ denotes the electron spin, $p$ and $L$ are the momentum and the orbital angular momentum respectively, $V_{\text {nuc }}$ indicates the potential near the nucleus, and $V_{\text {surface }}$ is the potential at the surface. For heavy atoms and distances $r<a_{B} / Z=R_{0}, \quad V_{\text {nuc }}$ can be approximated by $V_{\text {nuc }}(r)=-Z e^{2} / r$, where $Z$ is the nucleus charge and $a_{B}$ is the Bohr radius. From this expression we see that the splitting originates from the potential gradients and we can also separate the different contributions coming from the nucleus and surface region. The wave function of a $p_{z}$-type Shockley surface state can be written in separate coordinates as

$$
\Psi\left(x^{\prime}, y^{\prime}, z^{\prime}\right)=\phi\left(y^{\prime}\right) \phi\left(x^{\prime}\right) \varphi\left(z^{\prime}\right),
$$

where $\varphi\left(z^{\prime}\right)$ is already defined in Eq. (7). The surface state wave function in the case of the flat $\mathrm{Au}(111)$ surface is obtained by substituting $\phi\left(x^{\prime}\right) \phi\left(y^{\prime}\right)=e^{i k_{x^{\prime}} x^{\prime}} e^{i k_{y^{\prime}} y^{\prime}}$ in Eq. (15). The energy splitting given by the first order perturbation theory is then

$$
\Delta E_{\text {flat }}=\frac{\hbar^{2} \sqrt{k_{x^{\prime}}^{2}+k_{y^{\prime}}^{2}}}{2 m^{* 2} c^{2}}|2 \pi I+J|,
$$

where $I$ and $J$ are the integrals representing the nuclear region and surface contributions, respectively, and $m^{*}$ is the effective mass of the surface state band. From this equation we can already deduce the linear dependence of the splitting on the momentum parallel to the surface, as expected from both experiment and theory. The nuclear region contribution $I$ can be expressed in the following way: ${ }^{26}$

$$
\begin{aligned}
I= & \sum_{i} \frac{Z^{2} e^{2} k_{z^{\prime}}^{2} e^{-2 \lambda_{c}\left|z_{i}^{\prime}\right|} A_{c}^{2}}{8 S \lambda_{c}^{4} a_{B}}\left\{\left[\left(2 \lambda_{c}^{2} R_{0}^{2}+3\right) \sinh \left(\lambda_{c} R_{0}\right)\right.\right. \\
& \left.-2 \lambda_{c} R_{0}\left[2 \cosh \left(2 \lambda_{c} R_{0}\right)+1\right]\right\},
\end{aligned}
$$

where $S$ is the area of the unit cell of the (111) surface and $z_{i}^{\prime}$ indicates the atomic plane positions. Assuming the crystal is terminated by a constant potential $V_{0 z}$ in vacuum the surface contribution $J$ is obtained

$$
J \equiv J_{z^{\prime}}=V_{0 z}|\varphi(0)|^{2},
$$

where $V_{0 z}$ is taken from Ref. 28 (in Ref. 28 the potential step is given by $A_{10}$ ) and $\varphi(0)$ is the surface state wave function at the geometrical (jellium) edge of $\mathrm{Au}(111)$. With $V_{0 z}$ $=11 \mathrm{eV}$ and $|\varphi(0)|^{2}=0.18$ (Ref. 29) we obtain for the surface contribution to the SOC splitting at $E_{F} \Delta E_{\text {surf }}=7.6$ $\times 10^{-5} \mathrm{eV}$. This value, far too small to be observable experimentally, suggests that any correction introducing a more realistic description of the surface potential does not change qualitatively the obtained result. Hence the only important contribution to the SOC splitting of the surface state band results from the nuclear contribution $I$.

In the case of a vicinal surface the terrace surface state is a 1D QW state. Along the steps, we still have free-electronlike wave function $\phi\left(y^{\prime}\right)=e^{i k_{y^{\prime}} y^{\prime}}$, and perpendicular to the steps the wave function can be written in the form $\phi\left(x^{\prime}\right)$ $=A_{x^{\prime}} \cos \left(k_{x^{\prime}} x^{\prime}+\delta_{x^{\prime}}\right)$. With this wave function the SOC splitting is obtained as

$$
\Delta E_{\text {vicinal }}=\frac{\hbar^{2}\left|k_{y^{\prime}}\right|}{2 m^{* 2} c^{2}} \sqrt{J_{x^{\prime}}^{2}+\left(2 \pi I^{\prime}+J_{z^{\prime}}\right)^{2}},
$$

where $J_{x^{\prime}, z^{\prime}}$ and $I^{\prime}$ are integrals that represent the surface and nuclear contribution, respectively. The cancellation of the SOC splitting in the $x^{\prime}$ direction is clear from this equation, where $\Delta E_{\text {vicinal }}$ only depends on the momentum $k_{y^{\prime}}$ parallel to the terrace. Assuming the gradient of the electron potential at the step is qualitatively similar to that for the terrace we obtain for $J_{x^{\prime}}$ : 


$$
J_{x^{\prime}}=\left[V_{0 x^{\prime}}|\phi(0)|^{2}+V_{L x^{\prime}}|\phi(L)|^{2}\right] \int_{-z_{1}}^{\infty} \varphi^{2}\left(z^{\prime}\right) d z^{\prime},
$$

where $V_{0 x^{\prime}}, V_{L x^{\prime}}$ are the potential changes at the left and right edges of the terrace. The surface term $J_{z^{\prime}}$ is given by Eq. (18). Estimating $J_{x^{\prime}}$ one can note that all the potential changes at the surface and terraces are of the order of 1-10 $\mathrm{eV}$ and the integral in Eq. (20) is always smaller than 1. In addition, the nodes of the standing wave function at the edges of the terrace further decrease the step contribution term, giving a maximum limit for the total surface contribution of $<10^{-4} \mathrm{eV}$. Thus the spin-orbit splitting comes practically from the nuclear potential gradient. The reasons for that are the small potential gradients at the step edges and the surface-vacuum interface as well as the small overlap between the wave function and the step potentials at the step edges. For the integral $I^{\prime}$ in Eq. (19) we obtain

$$
I^{\prime}=a_{x^{\prime}} \sum_{n}\left|\phi\left(x_{n}^{\prime}\right)\right|^{2} I,
$$

where $a_{x^{\prime}}$ is the interatomic distance in the direction perpendicular to the step. For more than one atomic row per terrace the sum in Eq. (21) is equal to $1 / a_{x^{\prime}}$ and we finally obtain $I^{\prime}=I$, where $I$ is the integral of Eq. (17) for a flat surface. This result shows that the SOC splitting on terraces is equal to that obtained for a flat surface. As follows from Eq. (17), $I$ is proportional to the square of the nuclear charge $Z$, the wave function amplitude $A_{c}$, and the inverse interlayer spacing through $k_{z^{\prime}}$. It decays exponentially into the bulk and decreases when $\lambda_{c}$ increases. The exponential demonstrates that the largest contribution results from the nuclei of the surface layer. Before estimating $I$ we would like to note that the surface state wave function $\varphi\left(z^{\prime}\right)$ is averaged in the $x^{\prime}, y^{\prime}$ plane and the amplitude in the nuclear region is therefore overestimated. ${ }^{30}$ This behavior of $\psi\left(x^{\prime}, y^{\prime}, z^{\prime}\right)$ is especially important for the precise estimate of $I$ since the SOC splitting comes mostly from a very small sphere around the nucleus with radius $R_{0}=a_{B} / Z=0.0067 \AA^{-1}$. Using $\lambda_{c}$ $=0.14 \AA^{-1}$ obtained for an $x y$-averaged potential, ${ }^{29} \mathrm{Eq}$. (16) gives a value for $\Delta E \sim 1 \mathrm{eV}$, which is higher than the experimental value, as expected for an overestimated wave function. On the other hand, one can calculate the effect ive penetration in the nuclear region, $\lambda_{n}^{\text {eff }}$, from the experimental value of the splitting $\Delta E_{\text {exp }}=0.122 \mathrm{eV}$, obtaining a value of $\lambda_{n}^{\text {eff }}=0.30 \AA^{-1}$. This effective penetration would decrease in
$50 \%$ the probability density in the nuclear region, which is in agreement with other nonaveraged wave function calculations (see Figs. 2 and 11 in Ref. 30). Thus, we conclude that only the nuclear term of the potential gradient is important for the SOC and that, due to the damping of the surface state, only the first two or three layers contribute.

\section{CONCLUSIONS}

In summary, angle-resolved photoemission data of vicinal $\mathrm{Au}(232321)$ reveal one-dimensional behavior of the surface state due to confinement at the terraces, as previously found in $\mathrm{Au}(8 \mathrm{8}$ 7). Comparison of the QW energy levels of both samples with the case of an infinite potential well show that the transmission probability for an electron impinging on a step $|T|^{2} \sim 0$ in both cases.

The wave functions of these lateral QW states can be directly obtained from the angle-resolved photoemission intensity analysis. These wave functions are compared to the case of an infinite potential well. The good agreement again suggests no transmission of the electron through the step edges, as has been previously observed by STM for different noble metals. ${ }^{8,9}$

As a consequence of the 1D confinement the spin-orbit interaction becomes anisotropic: the energy splitting in the direction perpendicular to the steps is cancelled. This result is reproduced using first order perturbation theory, which gives insight into the nature and origin of the SOC .

Finally, surface umklapp or diffraction of the final states due to the step array is observed in the photoemission spectra. A Fourier analysis of the QW states at different photon energies shows the final state split by the superlattice wave vector $g=2 \pi / d$, as expected for diffraction from the steps. The alignment of the spectra for different photon energies along the [111] direction indicates that the penetration of the QW state on the surface is perpendicular to the terraces.

\section{ACKNOWLEDGMENTS}

E.V.C. thanks P.M. Echenique for fruitful discussions. A.Mu., F.J.G. de A., E.V.C., Y.M.K., and J.E.O. are supported by the Universidad del País Vasco (Grant No. 1/UPV/ EHU/00057.240-EA-8078/2000). V.R. and S.R. are supported by the CNRS-ULTIMATECH program, the CRIF and the Universite de Paris 7. A. Ma. was supported by Contract No. HPMF-CT-2000-00565. The experiments at the SRC were supported by NSF Grants No. DMR-0084402, DMR9704196, and DMR-9815416.
${ }^{1}$ F. J. Himpsel, J. E. Ortega, G. J. Mankey, and R. F. Willis, Adv. Phys. 47, 511 (1998).

${ }^{2}$ R. Nötzel and K. H. Ploog, Adv. Mater. 522, (1993); R. Nötzel, Z. Niu, M. Ramsteimer, H. P. Schönherr, A. Trampert, L. Däweritz, and K. H. Ploog, Nature (London) 392, 56 (1998); P. Segovia, D. Purdie, M. Hegsberger, and Y. Baer, ibid. 402, 504 (1999); P. Gambardella, A. Dellmeyer, K. Maiti, M. C. Malagoli, W. Eberhardt, K. Kern, and C. Carbone, ibid. 416, 301 (2002).
${ }^{3}$ J. E. Ortega, S. Speller, A. Bachmann, A. Mascaraque, E. G. Michel, A. Mugarza, A. Närmann, A. Rubio, and F. J. Himpsel, Phys. Rev. Lett. 84, 6110 (2000).

${ }^{4}$ A. Mugarza, A. Mascaraque, V. Pérez-Dieste, V. Repain, S. Rousset, F. J. García de Abajo, and J. E. Ortega, Phys. Rev. Lett. 87, 107601 (2001).

${ }^{5}$ J. E. Ortega, A. Mugarza, V. Repain, S. Rousset, V. Pérez-Dieste, and A. Mascaraque, Phys. Rev. B 65, 165413 (2002). 
${ }^{6}$ F. Baumberger, T. Greber, and J. Osterwalder, Phys. Rev. B 64, 195411 (2001).

${ }^{7}$ X. Y. Wang, X. J. Shen, and R. M. Osgood, Jr., Phys. Rev. B 56, 7665 (1997).

${ }^{8} \mathrm{Ph}$. Avouris and I.-W. Lyo, Science 264, 942 (1994).

${ }^{9}$ L. Bürgi, O. Jeandupeux, A. Hirstein, H. Brune, and K. Kern, Phys. Rev. Lett. 81, 5370 (1998).

${ }^{10}$ M. Giesen and T. L. Einstein, Surf. Sci. 449, 191 (2000).

${ }^{11}$ V. Repain, J. M. Berroir, B. Croset, S. Rousset, Y. Garreau, V. H. Etgens, and L. Lecoeur, Phys. Rev. Lett. 84, 5367 (2000).

${ }^{12}$ T. Abukawa, M. Sasaki, F. Hisamatsu, T. Goto, T. Kinoshita, A. Kakizaki, and S. Kono, Surf. Sci. 325, 33 (1995).

${ }^{13}$ R. Paniago, R. Matzdorf, G. Meister, and A. Goldmann, Surf. Sci. 336, 113 (1995).

${ }^{14}$ F. Reinert, G. Nicolay, S. Schmidt, D. Ehm, and S. Hüfner, Phys. Rev. B 63, 115415 (2001).

${ }^{15}$ S. LaShell, B. A. McDougall, and E. Jensen, Phys. Rev. Lett. 77, 3419 (1996).

${ }^{16}$ J. Kliewer, R. Berndt, E. V. Chulkov, V. M. Silkin, P. M. Echenique, and S. Crampin, Science 288, 1399 (2000).

${ }^{17}$ Note that, in the Fabry-Pérot model of Ref. 9 absorption is included, thus the reflectivity $|R|^{2}<1$ obtained by this group is still consistent with no transmission throughout the steps. This is reinforced by the fact that they obtained a phase $\varphi=-\pi$, a condition implying total reflectivity if absorption is not taken into account.

${ }^{18}$ M. F. Crommie, C. P. Lutz, and D. M. Eigler, Science 262, 218
(1993).

${ }^{19}$ E. J. Heller, M. F. Crommie, C. P. Lutz, and D. M. Eigler, Nature (London) 369, 464 (1994).

${ }^{20}$ S. Crampin, N. H. Boon, and J. E. Inglesfield, Phys. Rev. Lett. 73, 1015 (1994).

${ }^{21}$ G. Hörmandinger, and J. B. Pendry, Phys. Rev. B 50, 18607 (1994).

${ }^{22}$ Value obtained from LDA-PW band calculations by Angel Rubio.

${ }^{23}$ M. Henzeler, Surf. Sci. 19, 159 (1970).

${ }^{24}$ A. Mugarza, J. E. Ortega, and F. J. García de Abajo, cond-mat/0208254 (unpublished).

${ }^{25}$ The case of spin-orbit splitting of standing waves extending from a single step on a flat surface along the normal to the step was studied by Petersen and Hedegård (Ref. 27), who showed that the spin-orbit splitting cannot be observed in surface state standing waves at the Fermi level with the Fourier transform STM method. Here we give a more transparent and complete insight of this problem.

${ }^{26}$ Yu. M. Koroteev, E. V. Chulkov, and P. M. Echenique (unpublished).

${ }^{27}$ L. Petersen and P. Hedegård, Surf. Sci. 459, 49 (2000).

${ }^{28}$ E. V. Chulkov, V. M. Silkin, and P. M. Echenique, Surf. Sci. 437, 330 (1999).

${ }^{29}$ E. V. Chulkov, V. M. Silkin, and M. Machado, Surf. Sci. 482-485, 693 (2001).

${ }^{30}$ E. V. Chulkov, V. M. Silkin, and E. N. Shirykalov, Surf. Sci. 188, 287 (1987). 